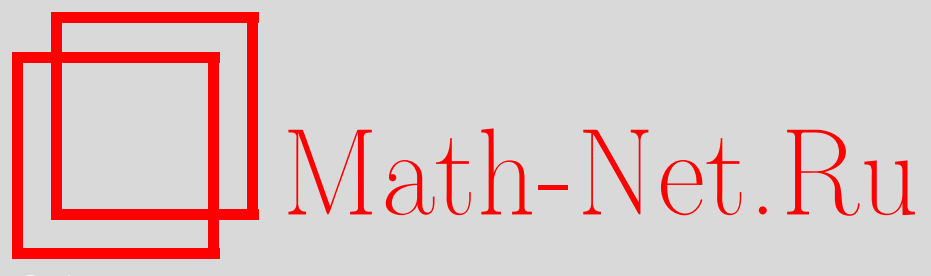

А. Е. Миронов, Т. Е. Панов, Гамильтоново-минимальные лагранжевы подмногообразия в торических многообразиях, УМН, 2013, том 68, выпуск 2, 203-204

DOI: https://doi.org/10.4213/rm9516

Использование Общероссийского математического портала Math-Net.Ru подразумевает, что вы прочитали и согласны с пользовательским соглашением http://www . mathnet.ru/rus/agreement

Параметры загрузки:

IP : 54.237 .59 .107

26 апреля 2023 г., 15:30:19

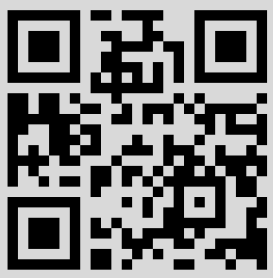




\title{
Гамильтоново-минимальные лагранжевы подмногообразия в торических многообразиях
}

\author{
А. Е. Миронов, Т. Е. Панов
}

Понятие гамильтоновой минимальности (Н-минималъности) для лагранжевых подмногообразий является симплектическим аналогом минимальности в римановой геометрии. Лагранжево погружение называется $H$-минимальным, если вариации его объема вдоль всех гамильтоновых векторных полей равны нулю. Это понятие было введено в работе О [1] в связи со знаменитой гипотезой Арнолъда о числе неподвижных точек гамильтонова симплектоморфизма.

В работах [2] и [3] авторами было введено и изучено семейство $H$-минимальных лагранжевых подмногообразий в $\mathbb{C}^{m}$, происходящих из пересечений вещественных квадрик. Здесь мы обобщаем эту конструкцию, определяя $H$-минимальные подмногообразия в торических многообразиях.

Начальными данными является пересечение $m-n$ эрмитовых квадрик в $\mathbb{C}^{m}$ :

$$
\mathscr{Z}=\left\{\boldsymbol{z}=\left(z_{1}, \ldots, z_{m}\right) \in \mathbb{C}^{m}: \sum_{k=1}^{m} \gamma_{j k}\left|z_{k}\right|^{2}=\delta_{j} \text { для } j=1, \ldots, m-n\right\} .
$$

Мы предполагаем, что это пересечение непусто, невырождено и рационально; эти условия выражаются в терминах векторов-коэффициентов $\gamma_{k}=\left(\gamma_{1 k}, \ldots, \gamma_{m-n, k}\right)^{t} \in$ $\mathbb{R}^{m-n}$ следующим образом:

a) $\delta \in \mathbb{R}_{\geqslant}\left\langle\gamma_{1}, \ldots, \gamma_{m}\right\rangle$ (т. е. $\delta$ лежит в конусе, порожденном $\gamma_{1}, \ldots, \gamma_{m}$ );

b) если $\delta \in \mathbb{R}_{\geqslant}\left\langle\gamma_{i_{1}}, \ldots, \gamma_{i_{p}}\right\rangle$, то $p \geqslant m-n$;

c) векторы $\gamma_{1}, \ldots, \gamma_{m}$ порождают решетку $L$ полного ранга в $\mathbb{R}^{m-n}$.

При этих условиях $\mathscr{Z}$ является гладким $(m+n)$-мерным подмногообразием в $\mathbb{C}^{m}$, а

$$
T_{\Gamma}=\left\{\left(e^{2 \pi i\left\langle\gamma_{1}, \varphi\right\rangle}, \ldots, e^{2 \pi i\left\langle\gamma_{m}, \varphi\right\rangle}\right), \varphi \in \mathbb{R}^{m-n}\right\}=\mathbb{R}^{m-n} / L^{*}
$$

является $(m-n)$-мерным тором. Определим также

$$
D_{\Gamma}=\left(\frac{1}{2} L^{*}\right) / L^{*} \cong\left(\mathbb{Z}_{2}\right)^{m-n} .
$$

Заметим, что $D_{\Gamma}$ канонически вкладывается в качестве подгруппы в $T_{\Gamma}$.

Пусть $\mathscr{R} \subset \mathscr{Z}$ - подмножество вещественных точек:

$$
\mathscr{R}=\left\{\boldsymbol{u}=\left(u_{1}, \ldots, u_{m}\right) \in \mathbb{R}^{m}: \sum_{k=1}^{m} \gamma_{j k} u_{k}^{2}=\delta_{j} \text { для } j=1, \ldots, m-n\right\} .
$$

Мы "разнесем" подмножество $\mathscr{R}$ действием тора $T_{\Gamma}$, т. е. рассмотрим множество $T_{\Gamma}$-орбит, проходящих через $\mathscr{R}$. Более точно, рассмотрим отображение

$$
j: \mathscr{R} \times T_{\Gamma} \longrightarrow \mathbb{C}^{m}, \quad(\boldsymbol{u}, \varphi) \mapsto \boldsymbol{u} \cdot \varphi=\left(u_{1} e^{2 \pi i\left\langle\gamma_{1}, \varphi\right\rangle}, \ldots, u_{m} e^{2 \pi i\left\langle\gamma_{m}, \varphi\right\rangle}\right)
$$

и заметим, что $j\left(\mathscr{R} \times T_{\Gamma}\right) \subset \mathscr{Z}$. Группа $D_{\Gamma}$ диагонально действует на $\mathscr{R}_{\Gamma} \times T_{\Gamma}$; это действие свободно, будучи свободным на втором сомножителе. Факторпространство

$$
N=\mathscr{R} \times{ }_{D_{\Gamma}} T_{\Gamma}
$$

является $m$-мерным многообразием.

Теорема 1 [2]. Отображение $j: \mathscr{R} \times T_{\Gamma} \rightarrow \mathbb{C}^{m}$ индуцирует $H$-минимальное лагранжево погружение $i: N \leftrightarrow \mathbb{C}^{m}$.

Работа выполнена при поддержке РФФИ (грант № 12-01-92104-Я), фонда “Династия" и гранта 2010-220-01-077 Правительства РФ. Первый автор поддержан также грантами Президента РФ МД-5134.2012.1 и НШ-544.2012.1. Второй автор поддержан также грантами Президента РФ МД-111.2013.1 и НШ-4995-2012.1 и грантом РФФИ 11-01-00694.

DOI: $10.4213 / \mathrm{rm} 9516$ 
Факторпространство $\mathscr{Z} / \mathbb{T}^{m}$ по действию тора $\mathbb{T}^{m} \subset \mathbb{C}^{m}$ отождествляется с множеством неотрицательных решений системы линейных уравнений $\sum_{k=1}^{m} \gamma_{k} y_{k}=\delta$. Это множество описывается выпуклым $n$-мерным полиэдром

$$
P=\left\{\boldsymbol{x} \in \mathbb{R}^{n}:\left\langle\boldsymbol{a}_{i}, \boldsymbol{x}\right\rangle+b_{i} \geqslant 0 \text { для } i=1, \ldots, m\right\},
$$

где $\left(b_{1}, \ldots, b_{m}\right)$ - произвольное решение, а система векторов $\boldsymbol{a}_{1}, \ldots, \boldsymbol{a}_{m} \in \mathbb{R}^{n}$ транспонирована к базису решений однородной системы $\sum_{k=1}^{m} \gamma_{k} y_{k}=\mathbf{0}$. Мы называем $P$ ассоциированным полиэдром системы квадрик (1).

Пусть $\Lambda$ - решетка ранга $n$, порожденная векторами $\boldsymbol{a}_{1}, \ldots, \boldsymbol{a}_{m}$. Полиэдр (2) называется делъзантовым, если для любой вершины $\boldsymbol{x} \in P$ векторы $\boldsymbol{a}_{i_{1}}, \ldots, \boldsymbol{a}_{i_{k}}$, нормальные к гиперграням, сходящимся в вершине $\boldsymbol{x}$, образуют базис решетки $\Lambda$.

Теорема 2 [3]. Погружение $i: N \leftrightarrow \mathbb{C}^{m}$ является вложением тогда и толъко тогда, когда ассоциированный полиэдр $P$ дельзантов.

Теперь рассмотрим две системы квадрик

$$
\mathscr{Z}_{\Gamma}=\left\{\boldsymbol{z} \in \mathbb{C}^{m}: \sum_{k=1}^{m} \gamma_{k}\left|z_{k}\right|^{2}=\boldsymbol{c}\right\}, \quad \mathscr{Z}_{\Delta}=\left\{\boldsymbol{z} \in \mathbb{C}^{m}: \sum_{k=1}^{m} \delta_{k}\left|z_{k}\right|^{2}=\boldsymbol{d}\right\},
$$

$\gamma_{k}, \boldsymbol{c} \in \mathbb{R}^{m-n}, \delta_{k}, \boldsymbol{d} \in \mathbb{R}^{m-\ell}$, такие, что множества $\mathscr{Z}_{\Gamma}, \mathscr{Z}_{\Delta} u \mathscr{Z}_{\Gamma} \cap \mathscr{Z}_{\Delta}$ удовлетворяют условиям а)-c) выше. Предположим также, что полиэдры, ассоциированные с $\mathscr{Z}_{\Gamma}$, $\mathscr{Z}_{\Delta}$ и $\mathscr{Z}_{\Gamma} \cap \mathscr{Z}_{\Delta}$, дельзантовы.

Определим вещественные пересечения квадрик $\mathscr{R}_{\Gamma}, \mathscr{R}_{\Delta}$, торы $T_{\Gamma} \cong \mathbb{T}^{m-n}, T_{\Delta} \cong$ $\mathbb{T}^{m-\ell}$ и группы $D_{\Gamma} \cong \mathbb{Z}_{2}^{m-n}, D_{\Delta} \cong \mathbb{Z}_{2}^{m-\ell}$ как описано выше.

Рассмотрим торическое многообразие $V$, получаемое симплектической редукцией пространства $\mathbb{C}^{m}$ по действию тора, соответствующего первой системе квадрик: $V=\mathscr{Z}_{\Gamma} / T_{\Gamma}$. Это - кэлерово многообразие вещественной размерности $2 n$. Факторпространство $\mathscr{R}_{\Gamma} / D_{\Gamma}$ является множеством вещественных точек в $V$ и имеет размерность $n$. Рассмотрим подмножество в $\mathscr{R}_{\Gamma} / D_{\Gamma}$, задаваемое второй системой квадрик:

$$
\mathscr{S}=\left(\mathscr{R}_{\Gamma} \cap \mathscr{R}_{\Delta}\right) / D_{\Gamma}
$$

тогда $\operatorname{dim} \mathscr{S}=n+\ell-m$. Наконец, определим $n$-мерное подмногообразие в $V$ :

$$
N=\mathscr{S} \times_{D_{\Delta}} T_{\Delta} .
$$

Теорема 3. $N$ является $H$-минимальным лагранжевым подмногообразием в $V$.

ДокАЗАТЕльство. Пусть $\widehat{V}$ - результат симплектической редукции многообразия $V$ по действию тора, соответствующего второй системе квадрик, т. е. $\widehat{V}=\left(V \cap \mathscr{Z}_{\Delta}\right) / T_{\Delta}=$ $\left(\mathscr{Z}_{\Gamma} \cap \mathscr{Z}_{\Delta}\right) /\left(T_{\Gamma} \times T_{\Delta}\right)$. Это - торическое многообразие размерности $2(n+\ell-m)$. Его подмногообразие вещественных точек

$$
\widehat{N}=N / T_{\Delta}=\left(\mathscr{R}_{\Gamma} \cap \mathscr{R}_{\Delta}\right) /\left(D_{\Gamma} \times D_{\Delta}\right) \hookrightarrow\left(\mathscr{Z}_{\Gamma} \cap \mathscr{Z}_{\Delta}\right) /\left(T_{\Gamma} \times T_{\Delta}\right)=\widehat{V}
$$

является множеством неподвижных точек комплексного сопряжения, т. е. вполне геодезическим подмногообразием. В частности, $\widehat{N}$ - минимальное подмногообразие в $\widehat{V}$. Согласно [4; следствие 2.7], $N$ является $H$-минимальным подмногообразием в $V$.

\section{Список литературы}

[1] Yong-Geun Oh, Math. Z., 212:1 (1993), 175-192. [2] А. Е. Миронов, Матем. сб., 195:1 (2004), 89-102. [3] А. Е. Миронов, Т. Е. Панов, Функи. анализ и его прил., 47:1 (2013), 47-61. [4] Yuxin Dong, Nonlinear Anal., 67:3 (2007), 865-882.

А. Е. Миронов (А. Е. Mironov)

Институт математики им. С. Л. Соболева

E-mail: mironov@math.nsc.ru

T. Е. Панов (T. E. Panov)

МГУ им. М. В. Ломоносова;

ИППИ РАН; ИТЭФ

E-mail: tpanov@mech.math.msu.su
Представлено В. М. Бухштабером Принято редколлегией 24.01.2013 